\title{
SINGLE AND DOUBLE ELEMENT PIFA MIMO ANTENNA WITH DGS FOR 5G MOBILE COMMUNICATION
}

\author{
C. Supratha and S. Robinson \\ Department of Electronics and Communication Engineering, Mount Zion College of Engineering and Technology, India
}

\begin{abstract}
The Planar Inverted F Antenna (PIFA) is mostly used in mobile communication. In this article, proposed two element PIFA is designed for $5 G$ applications. The design process is illustrated in a systematic manner, initially single element square patch antenna with spiral slot is designed. For improving the performance of the antenna the Defected Ground Structure (DGS) is established. Subsequently the two elements Multiple Input Multiple Output (MIMO) antenna is designed. The square shaped ring slot introduced on the ground to improve the performance of the antenna. The single and double elements are operated at $3.3 \mathrm{GHz}$ and $3.2 \mathrm{GHz}$. The antenna height is kept as $1.1 \mathrm{~mm}$. The patch dimension is $5.4 \mathrm{~mm} \times 5.4 \mathrm{~mm}$. The overall performance of the antenna in terms of $S$ parameter, radiation pattern, envelope correlation coefficient (ECC) are investigated. It is so compact also, the simulated results are exhibited in proposed antenna is suitable for $5 G$ mobile communication.
\end{abstract}

\section{Keywords}

MIMO, Defected Ground Structure, FR4, 5G, PIFA

\section{INTRODUCTION}

In recent year rapid growth of mobile communication make a need of high data rate and channel capacity. These requirements are solved by the $5^{\text {th }}$ generation. $5 \mathrm{G}$ spectrum is mainly divided in two parts, there are above $6 \mathrm{GHz}$ and below $6 \mathrm{GHz}$ [1]. $5 \mathrm{G}$ required advanced antenna technologies. Multiple Input Multiple Output (MIMO) technology satisfies the 5th generation demand.

Multiple antennas are used in transmitter and receiver side to improve the signal quality, reliability and channel capacity. Two main challenges are designed in MIMO system, there are, (i) size of the antenna and (ii) mutual coupling between the antenna elements.

In this literature survey, size reduction problem and mutual coupling is discussed. Rowell et al. [2] reviewed, the Planar Inverted F-Antenna (PIFA) is most suitable for mobile communication because the size of the antenna is equal to the quarter wavelength and backward radiation is eliminated [2]. Various size reduction methods are reported to minimize the size of PIFA antennas such as spiral shaped inverted F antenna [3], Slotted radiators [4], Meandered patch [5] and etc. Slot on the patch and slotted ground is used for improving the quality factor and compactness of the antenna [6]. The parametric variation for optimizing the antenna [7] is attempted. Periodical grooves are introduced on the patch is designed to increase the electrical length of the antenna [8]. The main drawback of PIFA is narrow bandwidth. The bandwidth is improved by increasing width of shorting pin and feed plate moreover the L shaped and rectangular parasitic element is placed in between the shorting pin and feed plate [9]. Capacitor plate is attached with the patch to decrease the size of the antenna. The capacitor plate is placed in between the radiating patch and ground plane [10].

In PIFA MIMO antenna, the mutual coupling is occurred in between the antenna element. Various isolation techniques are used to reduce mutual coupling such as decouple network, parasitic element [16], and defected ground structure [12] and neutralization line [17]. Three types of feed are examined. The feeds are parallel feed, coplanar feed and orthogonal feed. In those feed, the coplanar feed is provide the low mutual coupling [11]. Instead of two antennas two isolated feed plates are placed perpendicular to each other [12]. Two shorting pins and partial ground is incorporated to improve the isolation [13]. From the above mentioned papers, it is noticed that the PIFA MIMO antenna size is large and also produce high mutual coupling. In order to mitigate the issue, the low profile PIFA MIMO antenna is proposed.

In this paper PIFA MIMO antenna is proposed with low profile. The single element and with DGS, the double element and with DGS are designed. The initial design is single patch antenna with spiral shaped slot for optimizing the resonant frequency. The five rectangular slots are introduced on the ground. Then two elements MIMO antenna designed on same side with DGS.

This paper arranged as follow: section 2 briefs the methodology of the design, section 3 illustrates the design of single and double element antenna, section 4 illustrates the simulation and the result and section 5 concludes the paper.

\section{METHODOLOGY CALCULATION \\ AND \\ DESIGN}

The flowchart of design methodology of proposed antenna is shown in Fig.1. The proposed antenna is designed on low cost FR4 substrate with relative permittivity of 4.4.

Initially, the antenna requirements such as dimension of the patch, type of material, feeding method and etc. are analyzed. In second stage the single antenna element is designed and the performance is analyzed. If the performance cannot acceptable; some antenna enhancement technique is used. Antenna enhancement technique likes as slot and slit. In third process MIMO antenna is designed and the performance can be evaluated.

\subsection{DESIGN CALCULATION}

The resonant frequency can be calculated by using following formula [14]. Consider width and length of the patch is $5.4 \mathrm{~mm}$ and $5.4 \mathrm{~mm}$ respectively. According to the calculation the proposed antenna resonates at $3.3 \mathrm{GHz}$.

$$
f=\frac{c}{4(W+L)}
$$


where,

$$
\begin{aligned}
& C=\text { speed of light }=3 \times 10^{8} \mathrm{~m} / \mathrm{s} \\
& W=\text { Width of radiating patch } \\
& L=\text { Length of the radiating patch }
\end{aligned}
$$

The thickness of the substrate is $0.8 \mathrm{~mm}$. A dielectric loss tangent of FR4 is 0.02 . The patch size is $5.4 \mathrm{~mm} \times 5.4 \mathrm{~mm}$. The patch height is $1.1 \mathrm{~mm}$ from the ground plane. In between the substrate and patch is filled with air. According to the calculation then single and double element PIFA MIMO is designed.

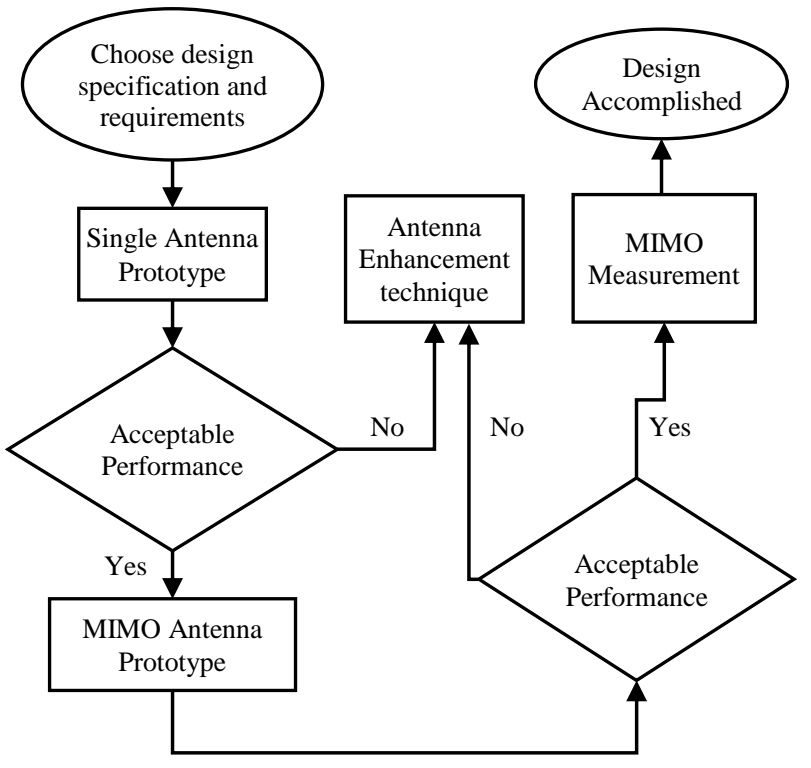

Fig.1. Flowchart of design methodology

\subsection{SINGLE ELEMENT WITHOUT DGS}

A single element of the proposed antenna is shown in Fig.2. Initially single element is designed. The proposed unit element is operated at $3.3 \mathrm{GHz}$.

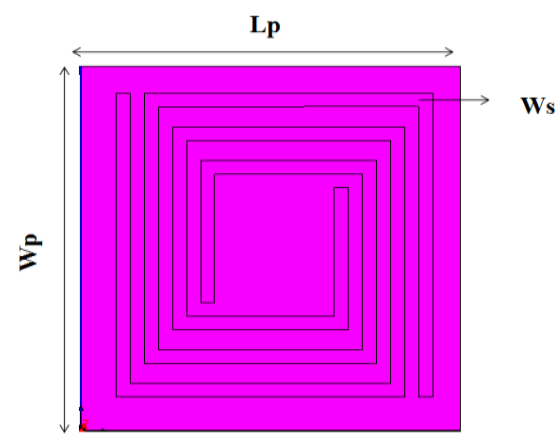

(a)

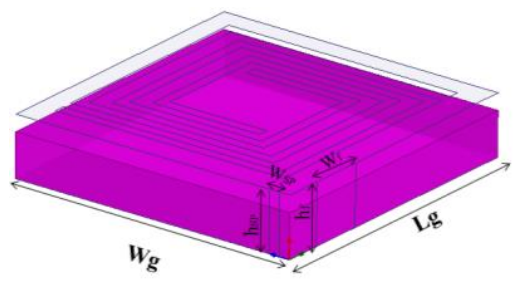

(b)

Fig.2. Schematic structure of single element PIFA MIMO antenna (a) Front view and (b) Side view
The antenna is fed by $50 \Omega$ microstrip line feed. The structural parameter of proposed antenna is illustrating in Table.1. The single element proposed antenna designed with spiral slot. Spiral

\begin{tabular}{|c|c|c|}
\hline Parameter & Symbols & Value $(\mathrm{mm})$ \\
\hline Patch length & $W_{p}$ & 5.8 \\
\hline Patch width & $L_{p}$ & 5.8 \\
\hline Ground Width & $W_{g}$ & 5.8 \\
\hline Ground length & $L_{g}$ & 5.8 \\
\hline Feed width & $W_{f}$ & 1.1 \\
\hline Feed height & $W_{h}$ & 1.1 \\
\hline Shorting pin width & $W_{s p}$ & 0.48 \\
\hline Shorting pin height & $L_{h}$ & 1.1 \\
\hline
\end{tabular}
slot reduce the electrical length of the antenna.

Table.1. Physical parameter of unit element (mm)

Fig.3. Simulated Return Loss response of single element PIFA MIMO antenna without DGS

The return loss $\left|S_{11}\right|$ of proposed antenna is shown in Fig.2. The return loss of proposed antenna is $-25.8 \mathrm{~dB}$ at $3.3 \mathrm{GHz}$. The various technique used for enhance the antenna performance. The most used techniques are DGS, parametric variation and slot. The parametric variation such as shoring pin dimension, feed dimension, patch and substrate dimension.

In this attempt, DGS technique for single and double element PIFA MIMO antenna is considered to minimize the mutual coupling.

\subsection{SINGLE ELEMENT WITH DGS}

The performance of the antenna is enhanced by introducing the DGS. The DGS is a simple technique to implement. The ground structure with DGS is shown in Fig.4. The five rectangular slots are introduced on the ground plane. Initially single slot is introduced on the ground plane. The number of slots in the ground plane is increased and their respective performances are investigated which are listed in Table.2. Hence, the five rectangular slots in a ground plane provide better performance. Hence it is accounted for further investigation. The rectangular slots suppress the unwanted wave from the ground plane and increase the performance of the antenna. Each slot length and width is $4.5 \mathrm{~mm}$ and $0.2 \mathrm{~mm}$. 


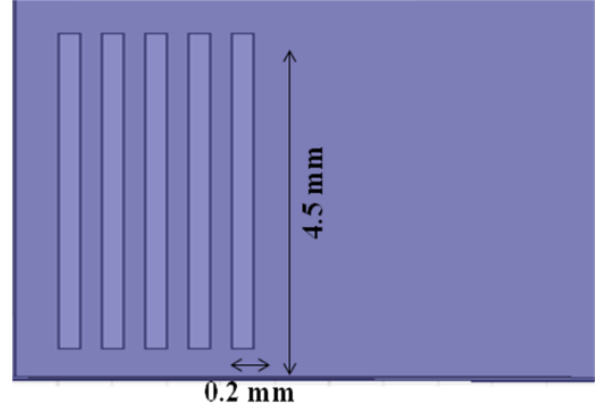

Fig.4. Defected ground structure in single element

Table.2. Impact of slot on double element PIFA MIMO antenna ground plane $(\mathrm{mm})$

\begin{tabular}{|c|c|c|}
\hline $\begin{array}{c}\text { Number } \\
\text { of slots }\end{array}$ & S11 & S22 \\
\hline 1 & $-12 \mathrm{~dB}$ & $-15 \mathrm{~dB}$ \\
\hline 2 & $-14 \mathrm{~dB}$ & $-16 \mathrm{~dB}$ \\
\hline 3 & $-16 \mathrm{~dB}$ & $-15 \mathrm{~dB}$ \\
\hline 4 & $-20 \mathrm{~dB}$ & $-13 \mathrm{~dB}$ \\
\hline 5 & $-22 \mathrm{~dB}$ & $-14 \mathrm{~dB}$ \\
\hline 6 & $-12 \mathrm{~dB}$ & $-13 \mathrm{~dB}$ \\
\hline
\end{tabular}

The return loss $\left|S_{11}\right|$ of proposed antenna is shown in Fig.5. The return loss of proposed antenna is increased at $-34.7 \mathrm{~dB}$ at 3.3 GHz after introducing the DGS.

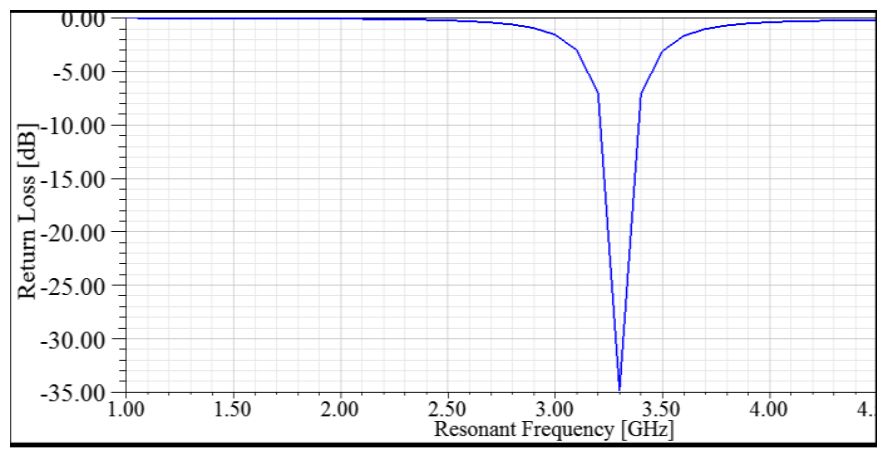

Fig.5. Simulated return loss response of single element PUFA MIMO with DGS

\subsection{DOUBLE ELEMENT WITHOUT DGS}

The design of double element MIMO antenna without DGS is shown in Fig.6. The antenna is designed by having two unit element antennas which are placed at same side. The distance between two antenna elements are varied by $\lambda, \lambda / 2, \lambda / 4, \lambda / 8$ and $\lambda / 16$ whose corresponding return loss performance are investigated which are listed in Table.3.

Among all, $\lambda / 8$ provides better performance than others; hence $\lambda / 8$ is accounted. Double element with lambda/ 8 distance design is shown in Fig.6. The return loss is reduced due to the mutual coupling. The signal is coupled one antenna to another. Hence the return loss of antenna 1 is reduced as $-20 \mathrm{~dB}$.

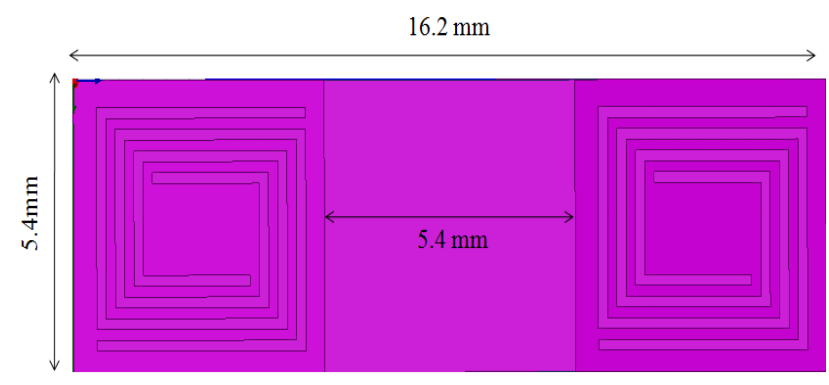

Fig.6. Schematic structure of double element PIFA MIMO antenna without DGS

The two antenna element is separated $5.4 \mathrm{~mm}$. The shorting pin and feed placed are adjacent side to each other. The length and width of double element PIFA MIMO antenna are $16.2 \mathrm{~mm}$ and $5.4 \mathrm{~mm}$ respectively. The return loss of PIFA MIMO antenna without DGS is shown in Fig.7.

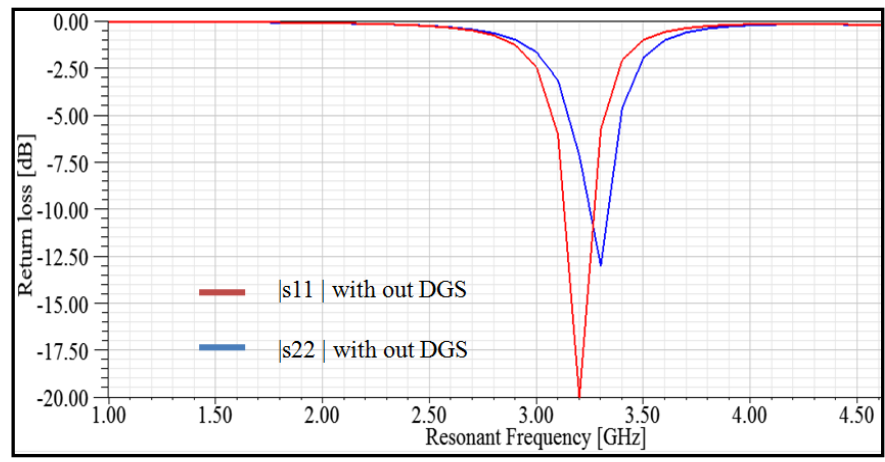

Fig.7. Return loss of double element PIFA MIMO antenna without DGS

\subsection{DOUBLE ELEMENT WITH DGS}

The five rectangular slots and square shaped ring slot are introduced on ground for improving the performance of the antenna. The defected ground structure is shown in Fig.8.

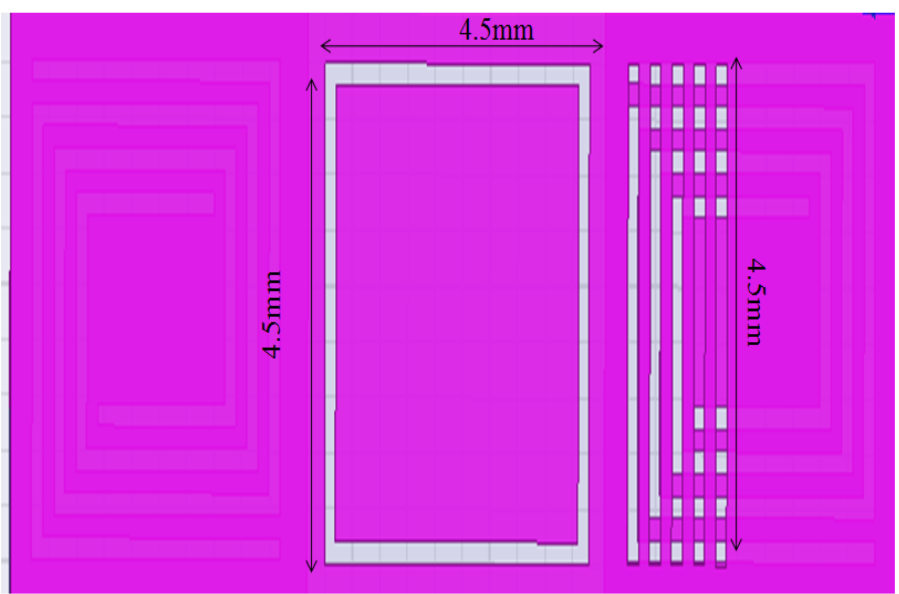

Fig.8. Schematic structure of double element PIFA MIMO antenna with DGS 
Table.3. Return loss performance of double element PIFA MIMO antenna (mm)

\begin{tabular}{|c|c|c|}
\hline Distance between the antenna & S11 & S22 \\
\hline Lambda & $-12 \mathrm{~dB}$ & $-15 \mathrm{~dB}$ \\
\hline Lambda/2 without DGS & $-14 \mathrm{~dB}$ & $-16 \mathrm{~dB}$ \\
\hline Lambda/4 without DGS & $-16 \mathrm{~dB}$ & $-15 \mathrm{~dB}$ \\
\hline Lambda/8 without DGS & $-20 \mathrm{~dB}$ & $-13 \mathrm{~dB}$ \\
\hline Lambda/16 without DGS & $-12 \mathrm{~dB}$ & $-15 \mathrm{~dB}$ \\
\hline Distance between the antenna & $\mathrm{S} 11$ & $\mathrm{~S} 22$ \\
\hline
\end{tabular}

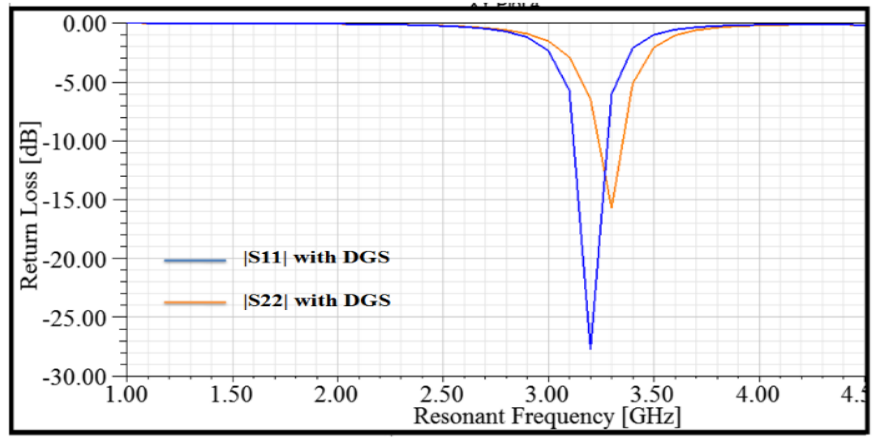

Fig.9. Simulated return loss response of double elements PIFA MIMO antenna with DGS

The return loss of proposed antenna is shown in Fig.8. The return loss of port 1 is $-27.75 \mathrm{~dB}$ at $3.2 \mathrm{GHz}$ for port 2 is $-15.77 \mathrm{~dB}$ at $3.3 \mathrm{GHz}$.

\section{RESULTS AND DISCUSSIONS}

The two elements PIFA MIMO with DGS antenna are designed. The distance between the antenna elements is kept as lambda/8.The isolation, radiation pattern and envelope correlation coefficient are simulated and reported here.

\subsection{ISOLATION}

Isolation is used for how much power is coupled between the adjacent antenna elements by using the structure of antenna elements. The Fig.10 shows the isolation curve of proposed four element MIMO antenna. The isolation calculated in between port 1 and 2 . The isolation is around $-12.50 \mathrm{~dB}$ at the resonant frequency is observed.

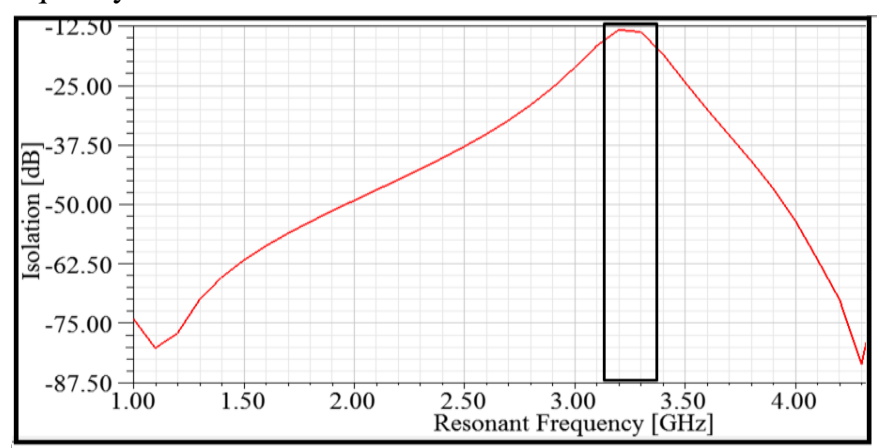

Fig.10. Simulated isolation response of double elements PIFA MIMO with DGS

\subsection{RADIATION CHARACTERISTICS}

The simulated radiation pattern of E-plane is presented in Fig.11. The simulated radiation pattern of proposed antenna at the frequency of $3.2 \mathrm{GHz}$ and $3.3 \mathrm{GHz}$ is investigated. The representation of the $2 \mathrm{D}$ radiation pattern of simulated result is unidirectional.

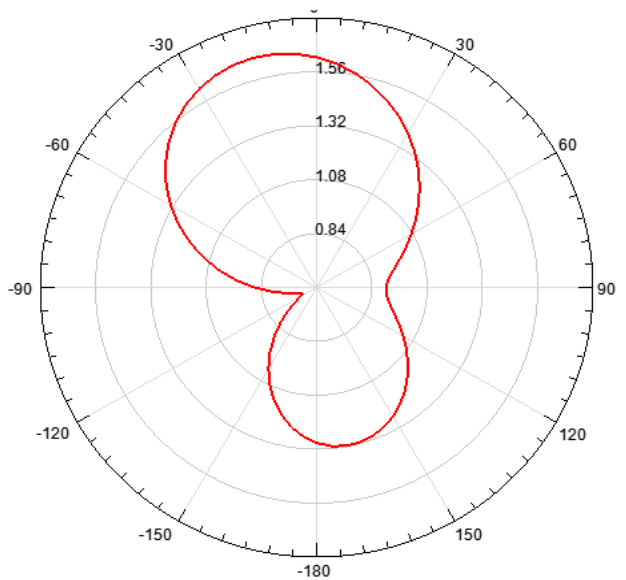

(a)

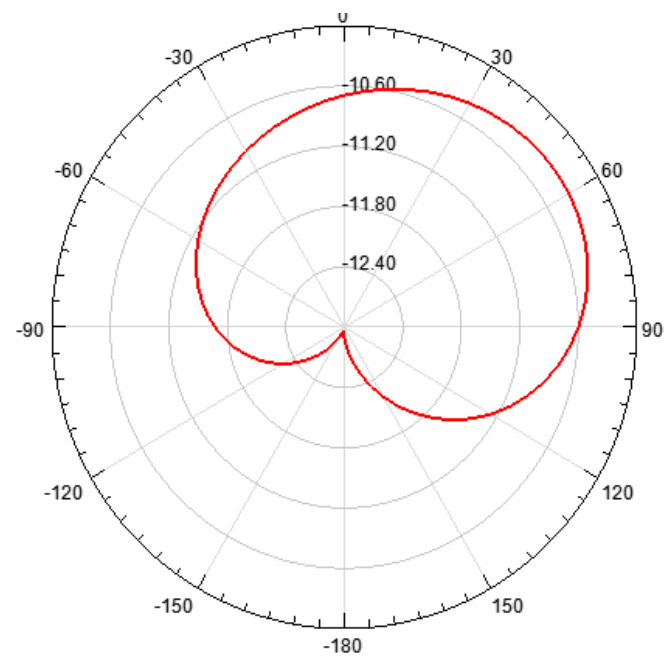

(b)

Fig.11. Radiation pattern of proposed PIFA MIMO antenna at E plane (a) $3.2 \mathrm{GHz}$ and (b) $3.3 \mathrm{GHz}$

\subsection{ENVELOPE CORRELATION COEFFICIENT (ECC)}

The capacity of proposed MIMO antenna is analyzed by ECC. The MIMO is necessary to obtain a low ECC. ECC compute the correlation between the branch signals received by the different element, and lower ECC signifies higher pattern diversity [15]. By assuming the proposed antenna as a lossless antenna in an isotropic scattering environment, the ECC between $i$ and $j$, is calculated. The ECC of two elements with DGS is shown in Fig. 12 (ECC at 0.071 at $3.3 \mathrm{GHz}$ ). 


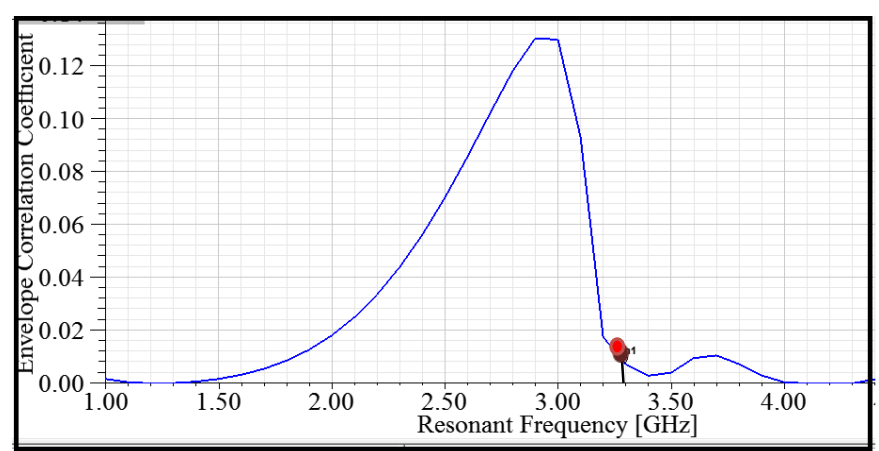

Fig.12. Simulated Envelope Correlation Coefficient response of two elements PIFA MIMO with DGS

\section{CONCLUSION}

In this paper two element PIFA MIMO antenna with DGS is proposed and designed. The functional characteristics of the proposed antenna namely return loss, isolation, radiation pattern and ECC are investigated. The overall size of the antenna is $16.2 \mathrm{~mm} \times 5.4 \mathrm{~mm} \times 1.1 \mathrm{~mm}$. The radiating patch size is $5.4 \mathrm{~mm} \times 5.4 \mathrm{~mm}$. The size of proposed antenna is small. The mutual couplings between the antennas elements are reduced by DGS. The ECC are less than 0.071. Here it meets the requirements for mobile applications.

\section{REFERENCES}

[1] David Stuart Muirhead, Muhammad Ali Imran and Kamran Arshad, "A Survey of the Challenges, Opportunities and Use of Multiple Antennas in Current and Future 5G Small Cell Substations", IEEE Transactions on Antennas and Propagation, Vol. 4, No. 1, pp. 2952-2964, 2016.

[2] Corbett Rowell and Edmund Y. Lam, "Mobile-Phone Antenna Design", IEEE Antennas and Propagation Magazine, Vol. 54, No. 4, pp. 14-34, 2012.

[3] Yu-Shin Wang, Ming- Chou Lee and Shyh-Jong, "Two PIFA- Related Miniaturized Dual Band Antennas", IEEE Transaction on Antennas and Propagation, Vol. 55, No. 3, pp. 805-811, 2007.

[4] Jaume Anguera, Ivan Sanz, Josep Mumbru and Carles Puente, "Multiband Handset Antenna with a Parallel Excitation of PIFA and Slot Radiators", IEEE Transactions on Antennas and Propagation, Vol. 58, No. 2, pp. 348-355, 2010.

[5] Abdelhakin Elouadih, Ahmed Qulad Said and Moha Mrabet Hassani, "Design and Simulation of a PIFA Antenna for The use in 4G Mobile Telecommunications Network",
International Journal of Communication Network and System Design, Vol. 6, pp. 325-332, 2013.

[6] Arnau Cabedo, Jaume Anguera, Cristina Picher, Miquel Ribo and Carles Puen, "Multiband Handset Antenna Combining a PIFA, Slots, and Ground Plane Modes", IEEE Transactions on Antennas and Propagation, Vol. 57, No. 9, pp. 2526-2533, 2009.

[7] Abdelhakin Elouadih, Ahmed Qulad Said and Moha Mrabet Hassani, "Design and Parametric Simulation of a Miniaturized PIFA Antenna for the PCS Band", Wireless Engineering and Technology, Vol. 4, No. 1, pp. 105-111, 2013.

[8] Nariman Firoozy and Mahmoud Shirazi, "Planar Inverted-F Antenna (PIFA) Design Dissection for Cellular Communication Application", Journal of Electromagnetic Analysis and Applications, Vol. 3, No. 1, pp. 406-411, 2011.

[9] H.T. Chattha, Y. Huang, M.K. Ishfaq and S.J. Boyes, "Bandwidth Enhancement Techniques for Planar Inverted F Antenna”, IET Microwaves, Antennas and Propagation, Vol. 5, No. 15, pp. 1872-1879, 2011.

[10] Dalia Mohammaed Nashaat, Hala K. Elsadek and Hani Ghali, "Single Feed Compact Quad-Band PIFA Antenna for Wireless applications", IEEE Transactions on Antennas and Propagation, Vol. 53, No. 8, pp. 2631-2625, 2005.

[11] Saqer Al Jaafreh, Yi Huang and Lei Xing, "Low Profile and Wideband Planar Inverted-F Antenna with Polarisation and Pattern Diversities", IET Microwaves, Antennas and Propagation, Vol. 10, No. 2, pp. 152-161, 2016.

[12] Hassan Tariq Chattha, Yi Huang, Stephen J. Boyes and Xu Zhu, "Polarization and Pattern Diversity-Based Dual-Feed Planar Inverted-F Antenna", IEEE Transactions on Antennas and Propagation, Vol. 60, No. 3, pp. 1532-1540, 2012.

[13] A. Jain, P.K. Verma and V.K. Singh, "Performance Analysis of PIFA based 4×4 MIMO Antenna", Electronics Letters, Vol. 48, No. 9, pp. 1-7, 2012.

[14] Naveen Kumar and Garima Saini, "A Novel Low Profile Planar Inverted F Antenna for Mobile Handsets", International Journal of Scientific and Research, Vol. 3, No. 3, pp. 10-12, 2013.

[15] A. Toktas and A. Akdagli, "Wideband MIMO Antenna with Enhanced Isolation for LTE, WiMAX and WLAN Mobile Handsets", Electronics Letters, Vol. 50, No. 10, pp. 723724, 2014.

[16] L. Minz and R. Garg, "Reduction of Mutual Coupling between Closely spaced PIFAs", Electronics Letters, Vol. 46, No. 6, pp. 663-672, 2010.

[17] Ahmed A. Naser, Khalil H. Sayidmaire and Jabir S. Aziz, "Compact High Isolation Meandered Line PIFA Antenna for LTE (Band Class-13) Handset Applications", Progress in Electromagnetic Research C, Vol. 67, pp. 153-164, 2016. 